\title{
Impulsive stabilization of delay difference equations and its application in Nicholson's blowflies model
}

\author{
Kaining $\mathrm{Wu}^{*}$ and Xiaohua Ding
}

* Correspondence: kainingwu@163. com

Department of Mathematics, Harbin Institute of Technology at Weihai, Weihai, 264209, China

\begin{abstract}
In this article, we consider the impulsive stabilization of delay difference equations. By employing the Lyapunov function and Razumikhin technique, we establish the criteria of exponential stability for impulsive delay difference equations. As an application, by using the results we obtained, we deal with the exponential stability of discrete impulsive delay Nicholson's blowflies model. At last, an example is given to illustrate the efficiency of our results.

Mathematics Subject Classification 2000: 39A30; 39A60; 39A10; $92 B 05$.
\end{abstract}

Keywords: impulsive, difference equation, exponential stability, stabilization, Nicholson's blowflies model

\section{Introduction}

Discrete systems exist in the word widely and most of them are described by the difference equations. The properties of difference equations, especially the stability and stabilization, were studied by many researchers, see [1-6] and the references therein.

As well known, in the practice, many systems are subject to short-term disturbances, these disturbances are often described by impulses in the modeling process, therefore the impulsive systems arise in many scientific fields and there are many works were reported on impulsive systems [7-16]. In those works, the stability study for the impulsive system is one of the research focuses, see [11-16].

In the study of stability, the Lyapunov function and Razumikhin method were used by many authors, see, for example, [6,17]. In [6], the Razumikhin technique was extended to the discrete systems. Although the stability of impulsive delay difference equations has been studied in some articles, for example, see [18], there are few article concerning on impulsive stabilization of delay difference equations. From the article [19], we know that the continuity is crucial in the proof of the stabilization theorem under the continuous situation. However, under the discrete situation, there is no continuity to be utilized. The loss of continuity puts difficulties in the way to get the stabilization theorem. The main aim of this article is to establish the criteria of impulsive stabilization for delay difference equations, using the Lyapunov function and Razumikhin method.

Biological models were studied by many authors, see [20-25] and the references therein. The stability of the positive equilibrium is a hot topic to be studied. In this

(c) 2012 Wu and Ding; licensee Springer. This is an Open Access article distributed under the terms of the Creative Commons Attribution License (http://creativecommons.org/licenses/by/2.0), which permits unrestricted use, distribution, and reproduction in any medium, provided the original work is properly cited. 
article, we also study the stabilization of an impulsive delay difference Nicholson's blowflies model. We take an unstable difference Nicholson's blowflies equation without impulses, then the impulsive effects are adopted and the criterion of stability is established for the impulsive Nicholson's blowflies model.

The rest of this article is organized as follows. In Section 2, we introduce our notations and definitions. Then in Section 3, we present a theorem of impulsive stabilization for delay difference equations. In Section 4, by using our result, we deal with the discrete impulsive delay Nicholson's blowflies equation. In Section 5, an example is given to illustrate the efficiency of our results.

\section{Preliminaries}

Let $\mathbb{R}$ denote the field of real numbers and $\mathbb{R}^{n}$ denote the $n$-dimensional Euclidean space. $\mathbb{N}$ and $\mathbb{Z}$ represent the natural numbers and the integer numbers respectively. For some positive integer $m, N_{-m}=\{-m, \ldots,-1,0\}$. Given a positive integer $m$, for any function $\phi: N_{-m} \rightarrow \mathbb{R}^{n}$, we define $\|\varphi\|_{m}=\max _{\theta \in N_{-m}}\{|\varphi(\theta)|\}$, where $|\cdot|$ presents the Euclidean norm.

We consider the following impulsive delay difference system:

$$
\left\{\begin{array}{l}
x(n+1)=f(n, x(n-m), x(n-m+1), \ldots, x(n)), n \neq \eta_{k}-1, \\
x\left(\eta_{k}\right)=\beta_{k} x\left(\eta_{k}-1\right)
\end{array}\right.
$$

where $x(n) \in \mathbb{R}^{n}, f: \mathbb{N} \times \underbrace{\mathbb{R}^{n} \times \cdots \times \mathbb{R}^{n}}_{m+1} \rightarrow \mathbb{R}^{n} . \beta_{k}$ is a constant for any $k \in \mathbb{N}$. The impulsive moments $\left\{\eta_{k}\right\}_{1}^{\infty}$ are natural numbers and satisfy $0=\eta_{0}<\eta_{1}<\cdots<\eta_{k}<\cdots, \eta_{k}$ $\rightarrow \infty$ as $k \rightarrow \infty$.

The following initial values are imposed on system (1):

$$
x(s)=\varphi(s), s \in N_{-m},
$$

where $\phi:[-m, 0] \rightarrow \mathbb{R}^{n}$ satisfies $\|\phi\|_{m}<\infty$.

We assume $f(n, 0,0, \ldots, 0) \equiv 0$, then systems (1) admits the trivial solution. We also assume that for any initial values $x(s)=\phi(s), s \in N_{-m}$, system (1) has a unique solution, denoted by $x(n, \phi)$.

Definition 1. [6] The trivial solution of (1) is said to be globally exponentially stable, if for any solution $x(n, \phi)$ with the initial data $x(n)=\phi(n), n \in N_{-m}$, there exist constants $\gamma>0$ and $M>0$ such that

$$
|x(n, \varphi)| \leq M\|\varphi\|_{m} e^{-\gamma n}, \forall n \in N_{-m} \cup \mathbb{N} .
$$

\section{Impulsive stabilization of delay difference equations}

In this section, we present the stabilization theorem of impulsive delay difference equations. By using the Razumikhin technique, we obtain the sufficient conditions to guarantee the exponential stability of system (1). Moreover, another criterion of exponential stability for system (1) is given, which does not depend on the Lyapunov function but just depends on the system function $f$, impulsive moments $\left\{\eta_{k}\right\}$ and the impulsive gain $\left\{\beta_{k}\right\}$. Some techniques we used in the proof of the stabilization theorem are motivated by [19]. 
Theorem 2. Assume there exist a positive function $V(n, x)$ and positive constants $c_{1}$, $c_{2}, p, \lambda, \alpha, \alpha>1$, such that

$C_{1}: c_{1}|x|^{p} \leq V(n, x) \leq c_{2}|x|^{p}$, for all $n \in N_{-m} \cup \mathbb{N}$ and $x \in \mathbb{R}^{n}$.

$C_{2}$ : If $n \neq \eta_{k}-1$, for any function $\phi: N_{-m} \cup \mathbb{N} \rightarrow \mathbb{R}^{n}$, the following inequality holds

$$
V(n+1, f(n, \varphi)) \leq(1+\lambda) V(n, \varphi(n))
$$

whenever $q V(n+1, \phi(n+1)) \geq V(n+s, \phi(n+s))$ for all $s \in N_{-m}$ where $q \geq e^{2 \lambda \alpha}$.

$C_{3}: V\left(\eta_{k}, \beta_{k}\left(\phi\left(\eta_{k}-1\right)\right)\right) \leq d_{k} V\left(\eta_{k}-1, \phi\left(\eta_{k}-1\right)\right)$, where $d_{k}>0$.

$C_{4}: \eta_{k+1}-\eta_{k} \leq \alpha, \ln d_{k}+\alpha \lambda<-\lambda\left(\eta_{k+1}-\eta_{k}\right)$.

Then, for any initial data $x(n)=\phi(n), n \in N_{-m}$, there exists a positive constant $C$, such that

$$
|x(n, \varphi)| \leq C\|\varphi\|_{m} e^{-\frac{\lambda}{p} n},
$$

that is, the trivial solution of system (1) is exponentially stable.

Proof. For the sake of simplicity, we write $V(n)=V(n, x(n))$.

Choose $M>1$, such that

$$
(1+\lambda) c_{2}\|\varphi\|_{m}^{p} \leq M\|\varphi\|_{m}^{p} e^{-\lambda \eta_{1}} e^{-\alpha \lambda}<M\|\varphi\|_{m}^{p} e^{-\lambda \eta_{1}} \leq q c_{2}\|\varphi\|_{m}^{p} .
$$

We claim that for any $n \in\left[\eta_{k}, \eta_{k+1}\right), k \in \mathbb{N}$,

$$
V(n) \leq M\|\varphi\|_{m}^{p} e^{-\lambda \eta_{k+1}} .
$$

First, we will show, when $n \in\left[0, \eta_{1}\right)$,

$$
V(n) \leq M\|\varphi\|_{m}^{p} e^{-\lambda \eta_{1}} .
$$

Obviously, when $n \in N_{-m}, V(n) \leq M\|\varphi\|_{m}^{p} e^{-\lambda \eta_{1}}$.

If (6) is not true, then there must be an $\bar{n} \in\left[0, \eta_{1}-1\right)$ and an $n^{*} \geq 0$ such that

$$
V(\bar{n}+1)>M\|\varphi\|_{m}^{p} e^{-\lambda \eta_{1}}, \quad V(n) \leq M\|\varphi\|_{m}^{p} e^{-\lambda \eta_{1}}, \quad n \leq \bar{n},
$$

and

$$
V\left(n^{*}\right) \leq c_{2}\|\varphi\|_{m}^{p}, \quad c_{2}\|\varphi\|_{m}^{p}<V(n) \leq M\|\varphi\|_{m}^{p} e^{-\lambda \eta_{1}}, \quad n^{*}<n \leq \bar{n} .
$$

It should be pointed out there may be a case $n^{*}=\bar{n}$, that is, there no $n$ satisfies the second segment of (7). If it is true, then for any $n \leq \bar{n}$, we have

$$
V(n) \leq c_{2}\|\varphi\|_{m}^{p} .
$$

Obviously, for any $s \in N_{-m}$,

$$
q V(\bar{n}+1)>q M\|\varphi\|_{m}^{p} e^{-\lambda \eta_{1}}>q c_{2}\|\varphi\|_{m}^{p} \geq V(\bar{n}+s) .
$$

From $C_{2}$ we get

$$
V(\bar{n}+1) \leq(1+\lambda) V(\bar{n}),
$$

that is

$$
\begin{aligned}
V(\bar{n}) & \geq \frac{1}{1+\lambda} V(\bar{n}+1)>\frac{1}{1+\lambda} M\|\varphi\|_{m}^{p} e^{-\lambda \eta_{1}} \\
& =\frac{e^{\alpha \lambda}}{1+\lambda} M\|\varphi\|_{m}^{p} e^{-\lambda \eta_{1}} e^{-\alpha \lambda} \\
& >M\|\varphi\|_{m}^{p} e^{-\lambda \eta_{1}} e^{-\alpha \lambda} \geq c_{2}\|\varphi\|_{m}^{p}
\end{aligned}
$$


which contradicts with (8), then there must be an $n$ such that the second segment of (7) holds.

When $n \in\left[n^{*}+1, \bar{n}\right]$, from (7),

$$
V(n+s) \leq M\|\varphi\|_{m}^{p} e^{-\lambda \eta_{1}}<q c_{2}\|\varphi\|_{m}^{p}<q V(n) .
$$

By virtue of condition $C_{2}$, when $n \in\left[n^{*}+1, \bar{n}\right]$,

$$
V(n) \leq(1+\lambda) V(n-1) .
$$

From the definitions of $\bar{n}$ and $n^{*}$, we have $V(\bar{n}+1) \geq V(\bar{n}+s)$ and $V\left(n^{*}+1\right) \geq V$ $\left(n^{*}+s\right)$, then we get

$$
q V(\bar{n}+1) \geq V(\bar{n}+s), \quad s \in N_{-m},
$$

and

$$
q V\left(n^{*}+1\right) \geq V\left(n^{*}+s\right), \quad s \in N_{-m} .
$$

Using condition $C_{2}$ and inequality (9), we obtain

$$
\begin{aligned}
V(\bar{n}+1) & \leq(1+\lambda) V(\bar{n}) \leq(1+\lambda)^{\bar{n}-n^{*}} V\left(n^{*}+1\right) \\
& \leq(1+\lambda)^{\alpha} V\left(n^{*}\right)<e^{\alpha \lambda} c_{2}\|\varphi\|_{m}^{p} .
\end{aligned}
$$

Since $V(\bar{n}+1)>M\|\varphi\|_{m}^{p} e^{-\lambda \eta_{1}}$, we get

$$
M\|\varphi\|_{m}^{p} e^{-\lambda \eta_{1}}<e^{\alpha \lambda} c_{2}\|\varphi\|_{m}^{p},
$$

which is in contradiction with (4), then (6) holds, that is (5) holds for $k=1$.

Now we assume (5) holds for $k=1,2, \ldots, h$ - 1, i.e. when $n \in\left[\eta_{k-1}, \eta_{k}\right), k=1,2, \ldots$, $h$,

$$
V(n) \leq M\|\varphi\|_{m}^{p} e^{-\lambda \eta_{k}}
$$

From condition $C_{3}$ and condition $C_{4}$,

$$
\begin{aligned}
V\left(\eta_{h}\right) & \leq d_{h} V\left(\eta_{h}-1\right) \leq d_{h} M\|\varphi\|_{m}^{p} e^{-\lambda \eta_{h}} \\
& \leq M\|\varphi\|_{m}^{p} e^{-\lambda \eta_{h+1}} e^{-\alpha \lambda} \leq M\|\varphi\|_{m}^{p} e^{-\lambda \eta_{h+1}} .
\end{aligned}
$$

Now we will show, when $n \in\left[\eta_{h}, \eta_{h+1}\right)$,

$$
V(n) \leq M\|\varphi\|_{m}^{p} e^{-\lambda \eta_{h+1}} .
$$

If (12) doesn't hold, there must be an $\bar{n} \in\left(\eta_{h}, \eta_{h+1}-1\right)$ and an $n^{*} \in\left[\eta_{h}, \bar{n}\right]$, such that

$$
V(\bar{n}+1)>M\|\varphi\|_{m}^{p} e^{-\lambda \eta_{h+1},} \quad V(n) \leq M\|\varphi\|_{m}^{p} e^{-\lambda \eta_{h+1}}, \quad n \in\left[\eta_{h}, \bar{n}\right],
$$

and

$$
V\left(n^{*}\right) \leq M\|\varphi\|_{m}^{p} e^{-\lambda \eta_{h+1}} e^{-\alpha \lambda}, \quad V(n)>M\|\varphi\|_{m}^{p} e^{-\lambda \eta_{h+1}} e^{-\alpha \lambda}, \quad n^{*}<n \leq \bar{n} .
$$

Now we claim $n^{*}<\bar{n}$. If it is not true, then $n^{*}=\bar{n}$. Since $q V(\bar{n}+1) \geq V(\bar{n}+s), s \in$ $N_{-m}$, from condition $C_{2}$, we get $V(\bar{n}+1) \leq(1+\lambda) V(\bar{n})$, that is 


$$
V\left(n^{*}\right)=V(\bar{n}) \geq \frac{1}{1+\lambda} V(\bar{n}+1) \geq \frac{e^{\lambda \alpha}}{1+\lambda} M\|\varphi\|_{m}^{p} e^{-\lambda \eta_{h+1}} e^{-\alpha \lambda}>M\|\varphi\|_{m}^{p} e^{-\lambda \eta_{h+1}} e^{-\alpha \lambda}
$$

which is in conflict with (13).

For $n \in\left[n^{*}+1, \bar{n}\right]$ and $s \in N_{-m}$,

$$
V(n+s) \leq M\|\varphi\|_{m}^{p} e^{-\lambda \eta_{h}}=e^{\lambda\left(\eta_{h+1}-\eta_{h}\right)} M\|\varphi\|_{m}^{p} e^{-\lambda \eta_{h+1}} \leq e^{2 \lambda \alpha} M\|\varphi\|_{m}^{p} e^{-\lambda \eta_{h+1}} e^{-\alpha \lambda} \leq q V(n) .
$$

Using condition $C_{2}$, we have

$$
V(n) \leq(1+\lambda) V(n-1), \quad n \in\left[n^{*}+1, \bar{n}\right],
$$

and, obviously,

$$
q V(\bar{n}+1) \geq V(\bar{n})
$$

then by virtue of condition $C_{2}$, we obtain

$$
V(\bar{n}+1) \leq(1+\lambda) V(\bar{n}) .
$$

Using the definition of $V\left(n^{*}\right)$, we can easily get

$$
q V\left(n^{*}+1\right)>V\left(n^{*}+s\right), s \in N_{-m} .
$$

Then, by virtue of condition $C_{2}$ we have

$$
V\left(n^{*}+1\right) \leq(1+\lambda) V\left(n^{*}\right)
$$

Consequently,

$$
\begin{aligned}
V(\bar{n}+1) & \leq(1+\lambda) V(\bar{n}) \leq(1+\lambda)^{\bar{n}-n^{*}} V\left(n^{*}+1\right) \\
& \leq(1+\lambda)^{\bar{n}-n^{*}+1} V\left(n^{*}\right) \leq(1+\lambda)^{\alpha} V\left(n^{*}\right) \\
& <e^{\alpha \lambda} M\|\varphi\|_{m}^{p} e^{-\lambda \eta_{h+1}} e^{-\alpha \lambda} \\
& =M\|\varphi\|_{m}^{p} e^{-\lambda \eta_{h+1}}<V(\bar{n}+1),
\end{aligned}
$$

which is a contradiction. Then (5) holds for $k=h+1$.

By induction, we know (5) holds for any $n \in\left[\eta_{k}, \eta_{k+1}\right), k \in \mathbb{N}$.

From condition $C_{1}$, for any $n \in\left[\eta_{k}, \eta_{k+1}\right), k \in \mathbb{N}$

$$
c_{1}|x(n, \varphi)|^{p} \leq V(n) \leq M\|\varphi\|_{m}^{p} e^{-\lambda \eta_{h+1}} \leq M\|\varphi\|_{m}^{p} e^{-\lambda n},
$$

that is

$$
|x(n, \varphi)| \leq\left(\frac{M}{c_{1}}\right)^{1 / p}\|\varphi\|_{m} e^{-\frac{\lambda}{p} n}
$$

which is the assertion.

Now we are on the position to state a corollary, which is another criterion of exponential stability for system (1). This criterion does not dependent on the Lyapunov function but just dependents on the system function, impulsive moments and impulsive gain.

Corollary 3. Assume that system (1) satisfies 
(1) for any $n \in \mathbb{N}$, there exist positive constants $u(n)$ and $a_{j}(n), j=0,1, \ldots, m$, such that

$$
|f(n, x(n-m), x(n-m+1), \ldots, x(n))| \leq u(n)|x(n)|+\sum_{j=0}^{m} a_{j}(n)|x(n-j)|
$$

and $\mu_{0}=\sup _{n \in \mathbb{N}}\{u(n)\}, \mu=\sup _{n \in \mathbb{N}}\left\{\sum_{j=0}^{m} a_{j}(n)\right\}$ are finite numbers.

(2) there exist positive constant $\lambda$, integer $\alpha>1$ and constant $q$, satisfying $q \geq e^{2 \lambda \alpha}$, such that $\mu q\left(\mu_{0}+\mu\right)<1$ and

$$
0<\frac{\mu_{0}^{2}+\mu_{0} \mu}{1-q \mu\left(\mu_{0}+\mu\right)}-1 \leq \lambda
$$

(3) $\eta_{k+1}-\eta_{k} \leq \alpha$ and $\ln d_{k}+\lambda\left(\eta_{k+1}-\eta_{\mathrm{k}}\right) \leq-\lambda \alpha$ where $d_{k}=\beta_{k}^{2}, k \in \mathbb{N}$.

Then, for any initial data $\phi(s), s \in N_{-m}$, the solution $x(n, \phi)$ of system (1) satisfies

$$
|x(n, \varphi)| \leq\|\varphi\|_{m} e^{-\frac{\lambda}{2} n},
$$

that is, the trivial solution of (1) is globally exponentially stable.

Proof. Let $c_{1}=c_{2}=1, p=2, V(n)=|x(n)|^{2}$ in Theorem 2. Under this situation, it is sufficient to verify the condition $C_{2}$ of Theorem 2. Using condition (1), Hölder inequality and the assumption $\left|x_{n+j}\right|^{2} \leq q\left|x_{n+1}\right|^{2}$, for $j \in N_{-m}$, if $n \neq \eta_{k}-1$, we can obtain

$$
\begin{aligned}
|x(n+1)|^{2} & =|f(n, x(n-m), x(n-m+1), \ldots, x(n))|^{2} \\
\leq & \left(u(n)|x(n)|+\sum_{j=0}^{m} a_{j}(n)|x(n-j)|\right)^{2} \\
& =u^{2}(n)|x(n)|^{2}+2 u(n)|x(n)|\left(\sum_{j=0}^{m} a_{j}(n)|x(n-j)|\right)+\left(\sum_{j=0}^{m} a_{j}(n)|x(n-j)|\right)^{2} \\
\leq & u^{2}(n)|x(n)|^{2}+u(n) \sum_{j=0}^{m} a_{j}(n)\left(|x(n-j)|^{2}+|x(n)|^{2}\right) \\
& \quad+\left(\sum_{j=0}^{m}\left(a_{j}(n)\right)^{\frac{1}{2}}\left(a_{j}(n)\right)^{\frac{1}{2}} \mid x(n-j)\right)^{2} \\
\leq & \quad u^{2}(n)|x(n)|^{2}+u(n) \sum_{j=0}^{m} a_{j}(n)\left(q|x(n+1)|^{2}+|x(n)|^{2}\right) \\
& \quad+\left(\sum_{j=0}^{m} a_{j}(n)\right)\left(\sum_{j=0}^{m} a_{j}(n) q|x(n+1)|^{2}\right) \\
\leq & u(n)\left(u(n)+\sum_{j=0}^{m} a_{j}(n)\right)|x(n)|^{2}+q\left(\sum_{j=0}^{m} a_{j}(n)\right)(u(n)+a(n))|x(n+1)|^{2} \\
\leq & \mu_{0}\left(\mu_{0}+\mu\right)|x(n)|^{2}+q \mu\left(\mu_{0}+\mu\right)|x(n+1)|^{2} .
\end{aligned}
$$


From condition (2) we have $q \mu\left(\mu_{0}+\mu\right)<1$, this yields

$$
|x(n+1)|^{2}-|x(n)|^{2} \leq\left(\frac{\left(\mu_{0}+\mu\right) \mu_{0}}{1-q \mu\left(\mu_{0}+\mu\right)}-1\right)|x(n)|^{2} \leq \lambda|x(n)|^{2} .
$$

That is,

$$
V(n+1)-V(n) \leq \lambda V(n) .
$$

This completes the proof. $\square$

\section{Application to discrete impulsive delay Nicholson's blowflies model}

Consider the discrete Nicholson's blowflies model with delay (see $[24,25]$ ):

$$
x(n+1)-x(n)=-c x(n)+a x(n-m) e^{-b x(n-m)}, \quad n=0,1, \ldots,
$$

where $c \in(0,1), a, b \in(0 ;+\infty)$ and $m \in \mathbb{N}$, together with the initial values

$$
x(n)=\varphi(n), \quad n \in N_{-m},
$$

where $\phi(n)>0, n \in N_{-m}$.

In view of the application of system (16) in practice, we only take an interest in the positive value of (16). When $c<a$, there is a unique positive equilibrium

$$
u^{*}=\frac{1}{b} \ln \frac{a}{c}
$$

In $[24,25]$, the authors studied the fold bifurcation and Neimark-Sacker bifurcation. For the convenience, we present the result in [25] as follows:

Lemma 4. Suppose that $c<a$ is satisfied and denotes

$$
a^{*}=c \exp \left(1+\frac{\left((1-c)^{2}+1-2(1-c) \cos \theta\right)^{\frac{1}{2}}}{c}\right)
$$

where $\theta$ is the solution of $\frac{\sin (m \theta)}{\sin ((m+1) \theta)}=\frac{1}{c}$, and $\theta \in\left(0, \frac{\pi}{m+1}\right)$,

(1) If $a<a^{*}$, then $u^{*}$ is asymptotically stable.

(2) If $a>a^{*}$, then $u^{*}$ is unstable.

Here, we assume that $a>a^{*}$ and consider a discrete impulsive Nicholson's blowflies model with delay:

$$
\left\{\begin{array}{l}
x(n+1)-x(n)=-c x(n)+a x(n-m) e^{-b x(n-m)}, \quad n \neq \eta_{k}-1, \\
x\left(\eta_{k}\right)=u^{*}+\beta_{k}\left(x\left(\eta_{k}-1\right)-u^{*}\right), \\
x(n)=\varphi(n), \quad n \in N_{-m}
\end{array}\right.
$$

where $\beta_{k} \in \mathbb{R}, \eta_{k}, k=1,2, \ldots$, are the instances of impulse effect, satisfying $0<\eta_{1}<\eta_{2}$ $<\ldots<\eta_{k}<\ldots$, and $\eta_{k} \rightarrow \infty$ as $k \rightarrow+\infty$. We suppose there exists a positive constant $\alpha$ such that $\eta_{k+1}-\eta_{k} \leq \alpha$.

Substituting $y_{n}=x_{n}-u^{*}$ into (17) yields

$$
\begin{cases}\gamma(n+1)=(1-c) y(n)+c\left(\gamma(n-m)+u^{*}\right) e^{-b y(n-m)}-c u^{*}, & n \neq \eta_{k}-1, \\ \gamma\left(\eta_{k}\right)=\beta_{k} \gamma\left(\eta_{k}-1\right), & n \in N_{-m} .\end{cases}
$$


Definition 5. We call the equilibrium $u^{*}$ of system (17) is exponentially stable, if the trivial solution of system (18) is exponentially stable.

It is easy to get that $\left(-u^{*},+\infty\right)$ is an invariant set of system (18). For $\{y(n)\} \subset\left(-u^{*}\right.$, $+\infty)$,

$$
\begin{aligned}
\mid f(n, y & (n), \ldots, \gamma(n-m)) \mid \\
& =\left|(1-c) \gamma(n)+c\left(\gamma(n-m)+u^{*}\right) e^{-b y(n-m)}-c u^{*}\right| \\
& \leq(1-c)|\gamma(n)|+c\left|\left(\gamma(n-m)+u^{*}\right) e^{-b y(n-m)}-c u^{*}\right| \\
& =(1-c)|\gamma(n)|+c\left|e^{-b \xi}(1-b(\xi+u))\right||\gamma(n-m)| \\
& \leq(1-c)|\gamma(n)|+c e^{b u^{*}}|\gamma(n-m)| \\
& =(1-c)|\gamma(n)|+a|\gamma(n-m)|,
\end{aligned}
$$

Where $\xi \in\left(-u^{*}, y(n-m)\right]$.

By using Corollary 3 , inequality (19) and noting $\eta_{k+1}-\eta_{k} \leq \alpha$, we can get the following corollary:

Corollary 6. Assume there exist constants $\lambda>0$, integer $\alpha>1$ and $q \geq e^{2 \lambda \alpha}$, such that the following inequalities hold

(1) $a q(1-c+a)<1$ and $0<\frac{(1-c)^{2}+a(1-c)}{1-a q(1-c+a)}-1 \leq \lambda$.

(2) $\ln \beta_{k}^{2}+\lambda\left(\eta_{k+1}-\eta_{k}\right) \leq-\lambda \alpha$.

Then, the positive equilibrium $u^{*}$ of (17) is exponentially stable.

Corollary 7. Suppose that $0<a(1-c+a)<1$ in system (17). Given a positive constant $\lambda$ and an integer $\alpha>1$ satisfying $\lambda<-\frac{1}{2 \alpha} \ln (a(1-c+a)), \eta_{k+1}-\eta_{k}<\alpha, k=1$, $2, \ldots$, and

$$
0<\frac{(1-c)^{2}+a(1-c)}{1-a e^{2 \alpha \lambda}(1-c+a)}-1 \leq \lambda
$$

If there exist constants $\left\{\beta_{k}\right\}_{k=1}^{\infty}$, such that

$$
\ln \beta_{k}^{2}<-2 \lambda \alpha,
$$

then, the positive equilibrium $u^{*}$ of (17) is exponentially stable.

Proof. Taking $q=e^{2 \lambda \alpha}$, noting $\eta_{k+1}-\eta_{k} \leq \alpha$ and by virtue of Corollary 6, we get the assertion directly.

Remark 8. Corollary 7 tells us, for any positive constant $\lambda$ satisfying $\lambda<-\frac{1}{2 \alpha} \ln (a(1-c+a))$, we can take an impulsive strategy $\left\{\eta_{k}\right\}_{k=1}^{\infty}$ and $\left\{\beta_{k}\right\}_{k=1}^{\infty}$, such that the equilibrium $u^{*}$ is exponentially stable, the exponential rate is less than $-\frac{\lambda}{2}$.

\section{Numerical experiments}

We take $a=0.03, b=0.5, c=0.001, m=1000$ in the system of (16), the equilibrium of Equation (16) is $u^{*}=6.8024$ and it is unstable [24,25] (see Figure 1), where the initial values are $\phi(n) \equiv 6$.

We adopt the impulsive control as follows:

Choose $\eta_{k}=3 k$, and then choose $\beta_{k}=e^{-0.3}$, take $\lambda=0.1$ and $q=2$.

The conditions of Corollary 6 are satisfied, then the positive equilibrium point of (16) is exponentially stable (see Figure 2 ), where the initial values are also $\phi(n) \equiv 6$. 

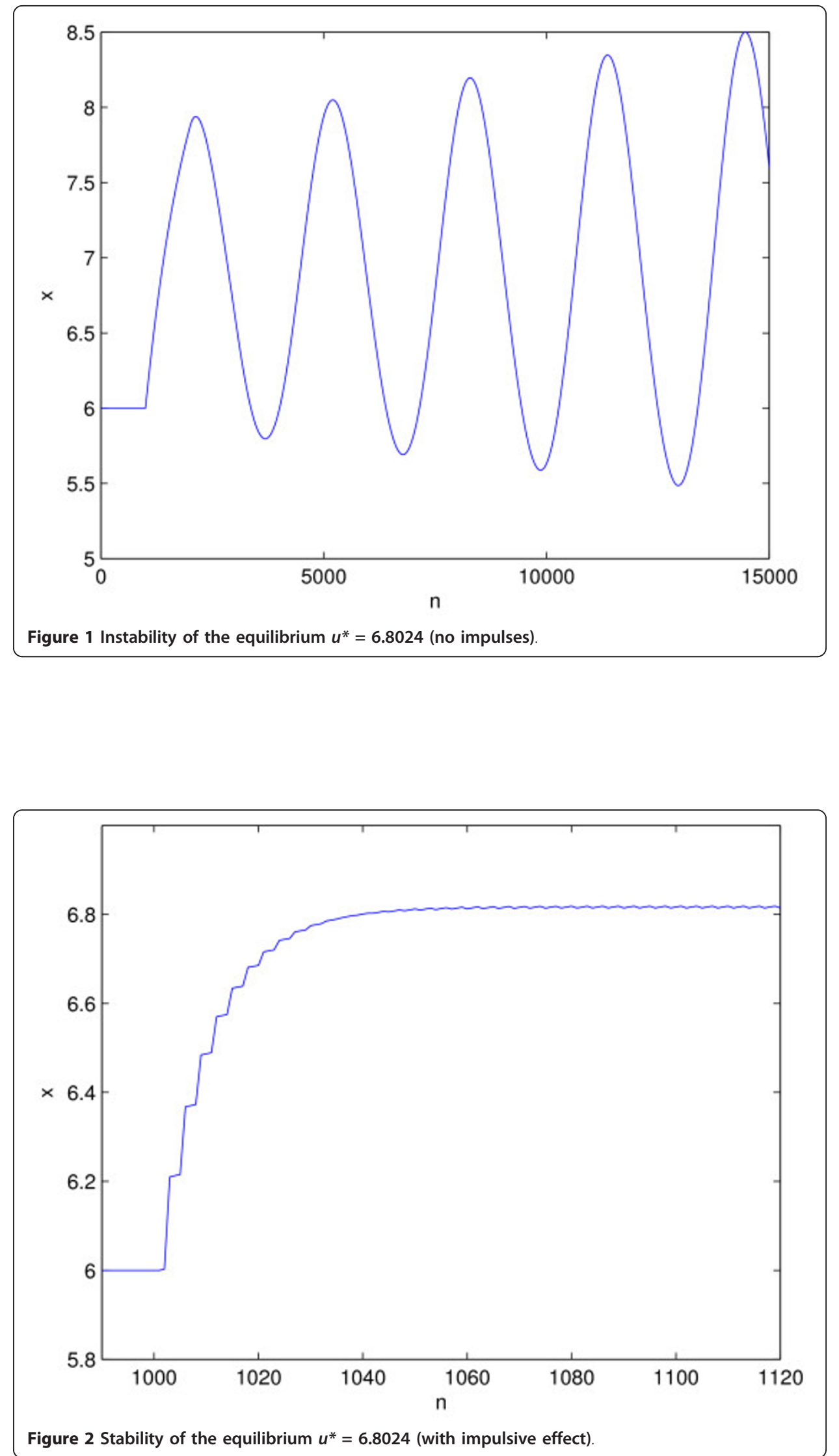


\section{Conclusion}

In this article, we established some global exponential stability criteria for impulsive delay difference systems by employing the Lyapunov function and Razumikhin technique. Using our result, we dealt with the discrete impulsive Nicholson's blowflies model. We obtained the sufficient conditions of exponential stability for the positive equilibrium of this model. At last, we presented an example to illustrated the e ciency of our results.

\section{Acknowledgements}

This work was supported by the National Natural Science Foundation of China under Grant 11026189 and by the Natural Scientific Research Innovation Foundation in Harbin Institute of Technology (HIT.NSRIF.201014).

\section{Authors' contributions}

Both authors contributed equally to the manuscript.

\section{Competing interests}

The authors declare that they have no competing interests.

Received: 28 March 2012 Accepted: 27 June 2012 Published: 27 June 2012

\section{References}

1. Paternoster, B, Shaikhet, L: Stability of equilibrium points of fractional difference equations with stochastic perturbations. Adv Diff Equ 2008, 21 (2008). doi:10.1155/2008/718408

2. Shaikhet, L: About an unsolved stability problem for a stochastic difference equation with continuous time. J Diff Equ Appl. 17, 441-444 (2011). doi:10.1080/10236190903489973

3. Zheng, B: Explicit conditions for stability of nonlinear scalar delay impulsive difference equation. Adv Diff Equ 2012, 17 (2012). doi:10.1155/2010/461014. doi:10.1186/1687-1847-2012-17

4. Xu, SY, Lamb, J, Yang, CW: Quadratic stability and stabilization of uncertain linear discrete-time systems with state delay. Syst Control Lett. 43, 77-84 (2001). doi:10.1016/S0167-6911(00)00113-4

5. Ferreira, C, Silva, FC: On the stabilization of linear discrete-time systems. Linear Alg Appl. 390, 7-18 (2004)

6. Liu, B, Marquez, HJ: Razumikhin-tpye theorems for discrete delay systems. Automatica. 43, 1219-1225 (2007). doi:10.1016/j.automatica.2006.12.032

7. De la Sen, M, Agarwal, RP, Ibeas, A, Alonso-Quesada, S: On the existence of equilibrium points, boundedness, oscillating behavior and positivity of a SVEIRS epidemic model under constant and SVEIRS epidemic model under constant and impulsive vaccination. Adv Differ Equ 2011, 32 (2011). doi:10.1155/2011/748608. doi:10.1186/1687-1847-2011-32

8. De la Sen, M, Agarwal, RP, Ibeas, A, Alonso-Quesada, S: On a generalized time-varying SEIR epidemic model with mixed point and distributed time-varying delays and combined regular and impulsive vaccination controls. Adv Diff Equ 2010, 42 (2010). doi:10.1155/2010/281612

9. Agarwal, RP, Karakoç, F: A survey on oscillation of impulsive delay differential equations. Comp Math Appl. 60, 1648-1685 (2010). doi:10.1016/j.camwa.2010.06.047

10. Agarwal, RP, Karakocc, F, Zafer, A: A survey on oscillation of impulsive ordinary differential equations. Adv Diff Equ 2010, 52 (2010). doi:10.1155/2010/354841

11. Niu, YJ, Liao, D, Wang, P: Stochastic asymptotical stability for stochastic impulsive differential equations and it is application to chaos synchronization. Commun Nonlinear Sci Numer Simul. 17, 505-512 (2012). doi:10.1016/j. cnsns.2011.07.011

12. Anokhin, A, Berezansky, L, Braverman, E: Exponential stability of linear delay impulsive differential-equations. J Math Anal Appl. 193, 923-941 (1995). doi:10.1006/jmaa.1995.1275

13. Berezansky, L, Braverman, E: On impulsive Beverton-Holt difference equations and their applications. J Diff Equ Appl. 193, 923-941 (1995)

14. Zhang, SR, Sun, JT, Zhang, Y: Stability of impulsive stochastic differential equations in terms of two measures via perturbing Lyapunov functions. Appl Math Comput. 218, 5181-5186 (2012). doi:10.1016/j.amc.2011.10.082

15. Li, CX, Sun, JT, Sun, RY: Stability analysis of a class of stochastic differential delay equations with nonlinear impulsive effects. J Frankl Inst. 347, 1186-1198 (2010). doi:10.1016/j.jranklin.2010.04.017

16. $\mathrm{Xu}, \mathrm{LG}, \mathrm{Xu}, \mathrm{DY}$ : Mean square exponential stability of impulsive control stochastic systems with time-varying delay. Phys Lett A. 373, 328-333 (2009). doi:10.1016/j.physleta.2008.11.029

17. Liu, B, Liu, XZ, Teo, K, Wang, Q: Razumikhin-type theorem on exponential stability of impulsive delay systems. IMA J Appl Math. 71, 47-61 (2006)

18. Zhu, W, Xu, DY, Yang, ZC: Global exponential stability of impulsive delay difference equations. Appl Math Comput. 181 65-72 (2006). doi:10.1016/j.amc.2006.01.015

19. Wang, Q, Liu, XZ: Impulsive sabilization of delay differential systems via the Lyapunov-Razumikhin method. Appl Math Lett. 20, 839-845 (2007). doi:10.1016/j.aml.2006.08.016

20. Bradul, N, Shaikhet, L: Stability of the positive point of equilibrium of Nicholson's blowflies equation with stochastic perturbations: numerical analysis. Discrete Dyn Natl Soc 2007, 25 (2007). doi:10.1155/2007/92959

21. Shaikhet, L: Lyapunov Functionals and Stability of Stochastic Difference Equations. Springer, New York/London/ Dordrecht/Heidelberg (2011) 
22. Berezansky, L, Braverman, E, Idels, L: Nicholson's blowflies differential equations revisited: Main results and open problems. Appl Math Model. 34, 1405-1417 (2010). doi:10.1016/j.apm.2009.08.027

23. Berezansky, L, Idels, L, Troib, L: Global dynamics of Nicholson-type delay systems with applications. Nonlinear Anal Real World Appl. 12, 436-445 (2011). doi:10.1016/j.nonrwa.2010.06.028

24. Ding, XH, Li, WX: Stability and bifurcation of numerical discretization Nicholson's blowflies equation with delay. Discret Dyn Natl Soc. 2006, 1-12 (2006)

25. Wang, CC, Wei, JJ: Bifurcation analysis on a discrete model of Nicholson's blowflies. J Diff Equ Appl. 14, 737-746 (2008). doi:10.1080/10236190701776035

doi:10.1186/1687-1847-2012-88

Cite this article as: Wu and Ding: Impulsive stabilization of delay difference equations and its application in Nicholson's blowflies model. Advances in Difference Equations 2012 2012:88.

Submit your manuscript to a SpringerOpen ${ }^{\circ}$ journal and benefit from:

- Convenient online submission

- Rigorous peer review

- Immediate publication on acceptance

- Open access: articles freely available online

- High visibility within the field

Retaining the copyright to your article

Submit your next manuscript at $\gg$ springeropen.com 